\title{
Expression of Phosphodiesterase (PDE) Isoenzymes in the Human Male and Female Urethra
}

This article was published in the following Dove Press journal: Research and Reports in Urology

\author{
Harrina E Rahardjo ${ }^{1,2}$ \\ Stefan Ückert ${ }^{2}$ \\ Andreas Bannowsky ${ }^{3}$ \\ Markus A Kuczyk ${ }^{2}$ \\ George T Kedia ${ }^{2,4}$ \\ 'Department of Urology, Universitas \\ Indonesia School of Medicine, Cipto \\ Mangunkusumo Hospital, Jakarta, \\ Indonesia; ${ }^{2}$ Hannover Medical School, \\ Division of Surgery, Department of \\ Urology \& Urological Oncology, \\ Hannover, Germany; ${ }^{3}$ Imland Hospital \\ $\mathrm{GmbH}$, Department of Urology, \\ Rendsburg, Germany; ${ }^{4}$ DIAKOVERE \\ $\mathrm{GmbH}$, Friederikenstift Lutheran \\ Hospital, Department of Urology, \\ Hannover, Germany
}

Purpose: Although it has been supposed that the NO/cyclic GMP system produces inhibitory signals to reduce the resistance of the bladder outlet and urethra during the micturition phase, little is known on the mechanisms controlling the function of urethral smooth muscle. The aim of the present study was to examine in the male and female urethra the expression of phosphodiesterase (PDE) isoenzymes, known as key proteins of the cyclic GMP/AMP signaling.

Methods: Urethral tissue was obtained from 4 female cadavers and 7 male patients (who had undergone gender reassignment surgery). The expression of mRNA encoding for PDE1A, 1B, 1C, 2A, 4B, 4D, 5A, 10A and 11A was investigated by means of real-time polymerase chain reaction. Western blot (WB) analysis was conducted to detect PDE isoenzymes.

Results: RT-PCR revealed relevant amounts of mRNA encoding for PDE1A, 2A, 4B, 5A, $10 \mathrm{~A}$ and $11 \mathrm{~A}$ in male and female urethral tissue. The expression of PDE1A, 2A, 4B and 10A was 2-fold higher in the female than in the male urethra, whereas the expression of PDE11A mRNA was 7-fold higher in the male tissue. In the WB experiments, immunosignals specific for PDE1A, PDE4A and 4B and PDE11A were of higher degree in the female than the male tissue specimens, while an almost equivocal expression of PDE2A, PDE5A and PDE10A was registered.

Conclusion: On the level of mRNA and function proteins, different patterns of expression of PDE isoenzymes were registered in human male and female urethra. Future studies may clarify whether inhibition of PDE isoenzymes is likely to facilitate the relaxation of the outflow region in both sexes.

Keywords: urethral smooth muscle, phosphodiesterase isoenzymes, cyclic adenosine monophosphate, nitric oxide/cyclic guanosine monophosphate

\section{Introduction}

The normal micturition cycle in females and males depends on the unimpaired function of the smooth musculature of the lower urinary tract (LUT), including the urethra. There is evidence that the urethra plays a role in maintaining continence in females, while, in males, the function of urethral smooth muscle (USM) has been attributed to transient changes in urethral resistance through relaxation and contraction during the active voiding phase. ${ }^{1,2}$ The male urethra is about $18-20 \mathrm{~cm}$ long and divided into an anterior and a posterior part. The wall of the urethra consists of four layers, including two layers of smooth musculature (an inner
Hannover Medical School, Department of Urology \& Urological Oncology, Hannover, 30623, Germany

Tel +495II 5323437

Fax +495II 5323481

Email sue_de_99@yahoo.de 
longitudinal and an outer circular layer). The length of the adult female urethra is approx. $4 \mathrm{~cm}$, it stretches from the bladder neck to the urethral orifice and is located behind the pubic symphysis. Transitional epithelial cells are lined along with the proximal part of the urethra, and convert into squamous epithelial cells lining most of the urethral length. In females, the striated muscle stretches the length of the urethra and consists almost exclusively of type I slow-twitch fibers (87\%) while in males the striated muscle stretches from the membranous urethra to the prostate and combines slow- and fast-twitch fibers $(65 \%$ vs $35 \%$, respectively). ${ }^{3,4}$ Experimental studies have indicated that the USM is under the control of the nitric oxide (NO)/cyclic GMP pathway, and a significance of cyclic GMP specific phosphodiesterase (PDE) isoenzymes, such as the PDE5, in this mechanism has been proposed. ${ }^{5,6}$ PDE enzymes are responsible for the degradation of the second messenger molecules cyclic GMP and cyclic AMP, thereby terminating the biological activity of these molecules. PDE inhibition has been proven to cause smooth muscle relaxation in various tissues of the male and female genitourinary tract and offers remarkable opportunities in the treatment of various diseases of the urogenital system..$^{7-9}$ It has been shown by means of the tissue bath technique that the selective PDE inhibitors vinpocetine, rolipram, sildenafil, vardenafil and tadalafil can reverse the tension induced via the activation of alphaadrenoceptors of isolated male USM. The relaxing effects of the said drugs were paralleled by an increase in cyclic AMP and/or cyclic GMP. ${ }^{10}$ In isolated human female urethra, the tonic contraction induced by noradrenaline was almost completely reversed by the PDE5 inhibitors sildenafil $(10 \mu \mathrm{M})$ and vardenafil $(30 \mu \mathrm{M}) .{ }^{11}$ By means of Western blotting and conventional immunohistochemistry, the expression of PDE1A, 4A, 4B and 5A was visualized in human urethral tissue. Smooth muscle bundles containing PDE proteins were seen innervated by nerve fibers characterized by the expression of the neuronal nitric oxide synthase (nNOS), calcitonin gene-related peptide (CGRP) and vasoactive intestinal polypeptide (VIP). ${ }^{12}$ Werkström et al reported that immunoreactivity specific for PDE5 was evident within urethral and vascular smooth muscle cells of the urethra of the female pig. ${ }^{11}$ However, up until today, studies on PDE enzymes in USM of males and females are scarce. Research efforts were limited to urethral tissue isolated from various animals (rabbit, rat, pig) or, in the case that human tissue specimens were utilized, focused on one PDE isoenzyme only, namely the cyclic GMP PDE5. ${ }^{11,13-15}$ The aim of the present study was to investigate by means of molecular biology (quantitative reverse transcriptase polymerase chain reaction $=$ qRT-PCR ) and Western blot analysis the expression of various PDE isoenzymes - PDE1 (isoforms A, B and C), PDE2A, PDE4 (isoforms $\mathrm{A} / \mathrm{B}$ and $\mathrm{D}$ ), PDE5A, PDE10A and PDE11A - in human female and male urethral tissue.

\section{Materials and Methods}

\section{Tissue Source}

The study protocol has been approved by the local Ethics Committee of the Hannover Medical School (Hannover, Germany) and it was in accordance with the Declaration of Helsinki. Urethral tissue was obtained from 4 female cadavers during forensic autopsies and 7 male patients who underwent male-to-female gender reassignment surgery. The mean age of both the female and male subjects was 48 years. If legally compulsory, informed consent was obtained from the subjects prior to the commencement of the study. Macroscopically normal urethral tissue was excised from the penile corpus spongiosum or the distal parts of the female urethras. Specimens were dissected free of fatty and connective tissue, immediately shockfrozen in liquid nitrogen and transported to the laboratory for further preparation.

\section{RT-PCR Experiments}

The expression of mRNA encoding for the PDE isoenzymes PDE1A, PDE1B and PDE1C $\left(\mathrm{Ca}^{2+} /\right.$ Calmodulindependent PDE), PDE2A (cyclic AMP PDE, activated by cyclic GMP), PDE4B and PDE4D (cyclic AMP-PDEs), PDE5A (cyclic GMP PDE), PDE10A and PDE11A (Dual Substrate PDEs) was analyzed by means of Real-time PCR using the TaqMan protocol. In brief, 1 to $5 \mu \mathrm{g}$ of total RNA was digested with DNase I and reverse transcribed into cDNA using the Superscript-II RT-PCR Kit (Invitrogen, Heidelberg, Germany). 2.5\% of the resulting cDNA (approx. $40 \mathrm{ng}$ ) was applied to amplification using the TaqMan Universal Master Mix (Eurogentec, Brussels, Belgium) and the following protocol: initial denaturation for $10 \mathrm{~min}$ at $94^{\circ} \mathrm{C}$. (denaturation for $15 \mathrm{sec}$ at $94^{\circ} \mathrm{C}$ ), annealing for $1 \mathrm{~min}$ at $60^{\circ} \mathrm{C}$, extension for $1 \mathrm{~min}$ at $72^{\circ} \mathrm{C}$, final extension for $10 \mathrm{~min}$ at $72^{\circ} \mathrm{C}, 40$ cycles. Fluorescence signals were detected using an ABI Prism 7700 device (Applied Biosystems, Framingham, USA). In order to exclude differences in total RNA concentration, partial 
degradation of RNA or an inappropriate cDNA synthesis, threshold cycle $(\mathrm{Ct})$ values were corrected for mRNA related to the housekeeping gene smooth muscle alphaactin. Expression was calculated using the formula relative expression $=2^{(20-\mathrm{dCt})}$ and given in arbitrary units (AU) $(\mathrm{dCt}=$ difference in $\mathrm{Ct}$ value between the gene of interest and the reference gene). The expression is displayed as the mean of three independent PCR amplification experiments for each primer.

\section{Western Blot Analysis}

Frozen urethral tissue was sectioned into slices, collected in tubes and homogenized in buffer (50 mM TRIS, $\mathrm{pH} 7.8$, containing protease inhibitor cocktail tablets and $150 \mathrm{mM}$ $\mathrm{NaCl}$ ) using a high-speed blender. Centrifugation was performed at $900 \mathrm{x} \mathrm{g}$ for $10 \mathrm{~min}$ to remove nonhomogenized material. Cytosolic supernatants were prepared and blots were blocked in 5\% skimmed milk in buffer. Specific antibodies directed against PDE1A, PDE2A, PDE4A and B, PDE5A, PDE10A and PDE11A (raised in rabbits, dilution 1:500) (FabGennix Inc., Frisco, TX, USA) were applied and Western blots performed as has been described previously and repeated in triplicate. ${ }^{12}$ The WB experiments were limited to those PDE isoenzymes which have been assumed by various authors to be of pharmacological significance in the lower urinary (and genital) tract of males and females. ${ }^{16}$

\section{Results qRT-PCR Analysis}

The efficiency of the various PCR reactions was close to $100 \%$, the calculated expression of mRNA specific for the PDE isoforms examined was only modestly influenced by the housekeeping gene used for normalization, indicating that mRNA levels of $\beta$-actin were almost similar in the tissues specimens applied to examination. The results provided no basis to assume that the time interval to tissue excision may have affected the results of the RT-PCR analysis. Potential contamination of the reaction mixtures was excluded using negative controls (no template and no amplification controls). RT-PCR revealed that mRNA encoding for all PDE isoenzymes, which were subject to this study, is expressed in the urethral tissues. Different magnitudes of mRNA expression were observed: RT-PCR revealed a predominant expression of mRNA transcripts encoding for PDE1A, PDE4B, PDE4D and PDE5A, whereas the expression of PDE1C, PDE2A and PDE10A was less pronounced and only negligible amounts of mRNA encoding for PDE1B were detected. The expression of PDE1A, 4B and 10A was approximately 2-fold higher in the female urethra compared to the male urethra. In contrast, the expression of mRNA encoding PDE11A was 7-fold higher in the male tissue. The results from the molecular biology studies are displayed in Figure $1(\mathrm{~A}-\mathrm{C})$.

\section{Western Blot Experiments}

In addition to the RT-PCR studies, the expression of PDE isoenzymes in the female and male urethra was also examined by Western blotting. Exposure of cytosolic supernatants prepared from rough homogenates of urethral tissue to anti-PDE1A, -PDE2A, -PDE4A, -PDE4B, -PDE5A, PDE10A or -PDE11A antibodies displayed clear bands without background shades within the expected molecular weight ranges (PDE1A: $65 \mathrm{kDa}$, PDE2A: $97 \mathrm{KD}$, PDE4A: 66 kDa, PDE4B: 66 kDa, PDE5A: 89-99 kDa, PDE10A: 90-93 kDa, PDE11A: 65-95 kDa). The degree of density of expression varied between the PDE isoenzymes: Signals specific for PDE2A, PDE5A and PDE10A were most pronounced in tissues taken from both sexes while the expression of protein accounting for PDE1A, PDE4A, PDE4B and PDE11A varied between females and males. In the WB experiments, signals specific for PDE2A, PDE5A and PDE10A were almost even in both the male and female urethras, in contrast, immunosignals specific for PDE1A, PDE4A, PDE4B and PDE11A were of higher degree in the female tissue specimens. In the female tissues, an almost equivocal expression of PDE2A, PDE4A, PDE10A and PDE11A was registered while in the male urethra, a similar degree of dense expression of PDE2A and PDE10A was noted. Exposure to anti-PDE11A antibodies resulted in a signal that appeared in abundance in the female urethra and was negligible in the male tissue samples. The results are displayed in Figure $2(A-G)$ and Table 1.

\section{Discussion}

Data from animal studies have provided extensive evidence that the urethra plays a functional role in maintaining continence and enabling coordinated micturition in dogs and cats, while, in man, the contraction and relaxation of urethral smooth muscle has been attributed to transient changes in urethral resistance during the voiding sequence. ${ }^{1}$ Most of the experiments to identify endogenous mediators contributing to the control of the outflow region were done using different animal models, therefore, 


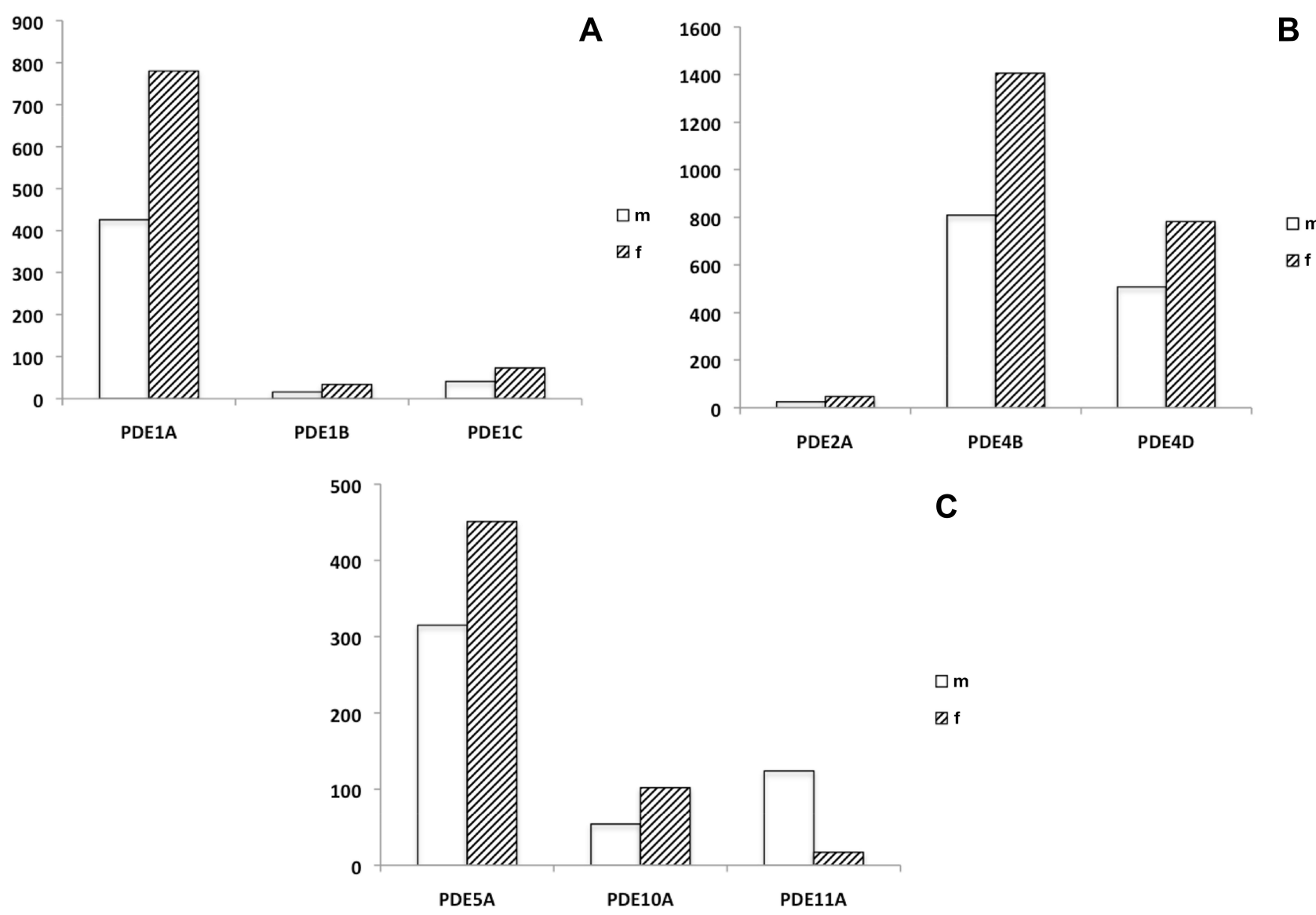

Figure I (A-C) Relative expression (as determined by means of qRT-PCR) of mRNA transcripts encoding for PDE isoenzymes IA, IB and IC (cyclic AMP-specific PDE, Ca ${ }^{2}$ `/calmodulin-dependent) (A), PDE2A (cyclic AMP PDE, cyclic GMP-dependent), PDE4B and 4D (cyclic AMP PDE) (B), PDE5A (cyclic GMP PDE), PDEIOA and IIA (I) (Dual Substrate PDEs) (C) in human male and female urethra. The (densitometric) degree of PDE-specific mRNA expression is given in arbitrary units (AU), related to the expression of the so-called house-keeping gene alpha-actin (known to be a characteristic marker protein of smooth musculature).

Abbreviations: $\mathrm{m}$, male; $\mathrm{f}$, female.

up until now, the pharmacology of the human urethra is still only poorly characterized. In contemporary urology, enhancement of cyclic nucleotide production brought about by NO-releasing drugs or selective inhibitors of PDE isoenzymes has become an interesting pharmacological option to influence the function of smooth musculature in the LUT. ${ }^{9}$ Studies on the expression and significance of key proteins of the NO/cyclic GMP or the cyclic AMP signalling pathways in the urethra have been conducted using mainly tissue from various animal species and only in seldom cases from humans. ${ }^{17-19}$ Very few studies have investigated PDE isoenzymes in the human urethra, these approaches were almost exclusively limited to the potential role of the cyclic GMP PDE5. Werkström et al investigated the effects of the PDE5 inhibitors sildenafil, vardenafil and tadalafil on isolated urethral smooth muscle, Fibbi et al showed the expression of PDE5 in human male prostatic urethra. ${ }^{11,20}$ The present study has revealed that, at the mRNA level, transcripts specifically encoding for PDE isoforms $1 \mathrm{~A}, 4 \mathrm{~B}, 4 \mathrm{D}$ and $5 \mathrm{~A}$ are predominantly expressed in urethral tissue of both sexes, whereas the expression of PDE1A and 4B was approximately 2 -fold higher in the female urethra compared to the male urethra. The amount of mRNA specific for PDE11A was clearly higher in male tissue. With regard to the mere expression of mRNA transcripts encoding for PDE isoenzymes, the findings are in agreement with a previous study demonstrating the expression of mRNA related to PDE1, PDE4 and PDE5 in human male USM. On the protein level, all PDE isoenzymes verified by means of qRT-PCR were also detected in the Western blot experiments. We found that signals specific for PDE2A, PDE5A and PDE10A were most pronounced in tissues taken from both sexes while immunosignals specific for PDE1A, PDE4A, PDE4B and PDE11A were of higher degree in the female urethra. By means of chromatographic and 

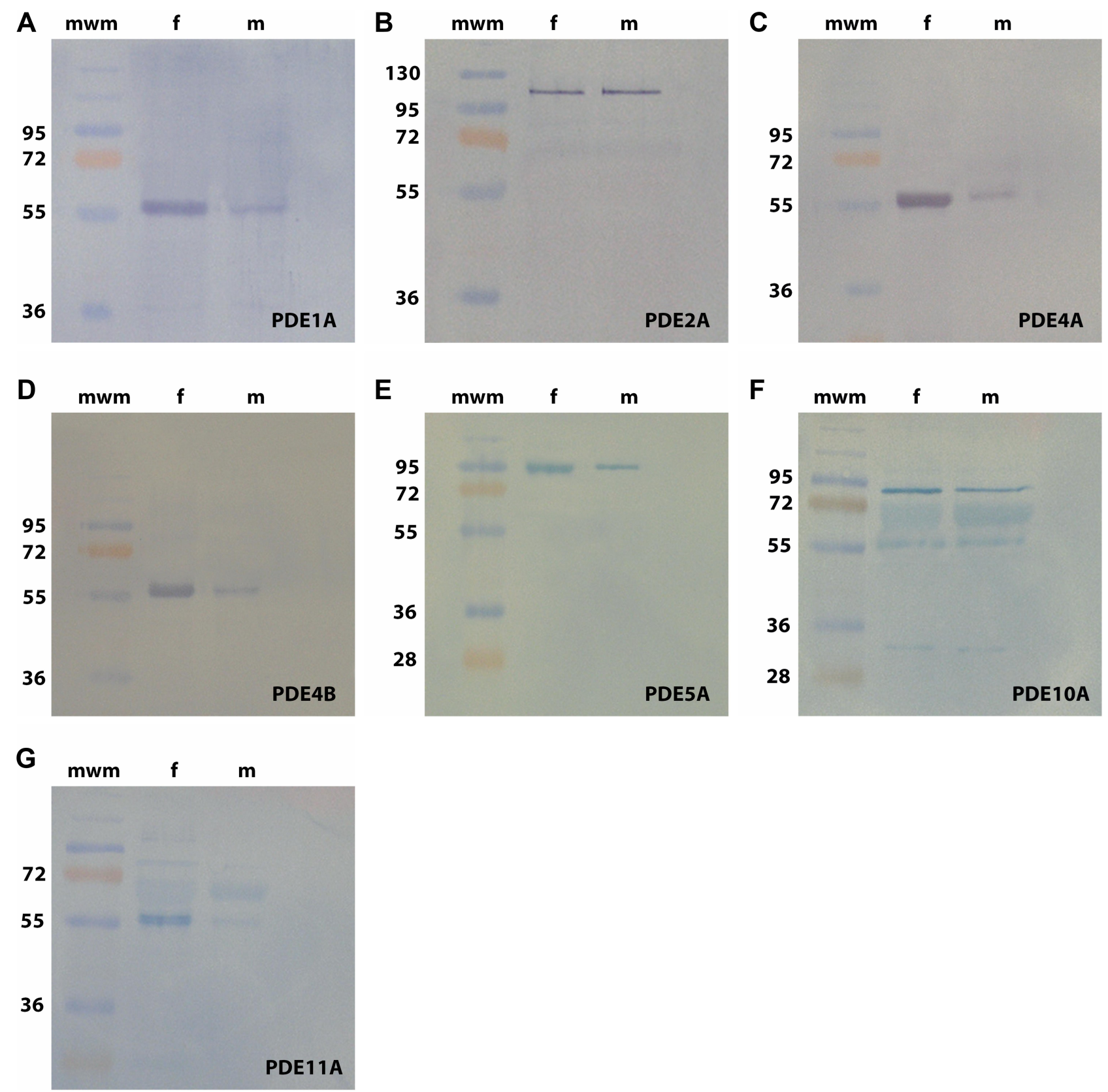

Figure 2 (A-G) Western blot analysis. Exposure to anti-PDEIA (A), -PDE2A (B), -PDE4A (C), -PDE4B (D), -PDE5A (E), -PDEI0A (F) and -PDEI IA (G) antibodies of cytosolic supernatants prepared from tissue specimens of the human male and female urethra displayed signals of the expected molecular weights for the PDE isoenzymes investigated: PDEIA: 65 kDa, PDE2A: 97 kDa, PDE4A/4B: 66 kDa, PDE5A: 89-99 kDa, PDEI0A: $90-93$ kDa, PDEI IA: 65-95 kDa. mwm = molecular weight marker (Page Ruler Plus ${ }^{\mathrm{TM}}$ Protein ladder, Fermentas $\mathrm{GmbH}$, St. Leon-Roth, Germany).

Abbreviations: $\mathrm{m}$, male; $\mathrm{f}$, female.

immunohistochemical methods, PDE1, PDE4 and PDE5 were also observed in the human urinary bladder of both sexes, with the cyclic GMP PDE5 being the most prominent isoenzyme in smooth muscle fibers of the bladder as well as in the endothelium and smooth muscle layer of vesicular deferential arteries. However, there is no evidence demonstrating substantial differences in the expression of PDE isoenzymes in detrusor smooth musculature of males and females. ${ }^{20-22}$ The apparent discrepancies in the expression of PDE enzymes on the level of mRNA and function proteins, for example, relatively high expression of mRNA encoding PDE1A vs mediocre to weak expression of PDE1 protein in the male urethra, might be due to post-transcriptional processing mechanisms so that steadystate mRNA levels do not necessarily correlate with the protein composition of the cell. ${ }^{23-25}$ The findings are also 
Table I Western Blot Experiments

\begin{tabular}{|l|c|c|}
\hline \multirow{2}{*}{ PDE Isoenzyme } & \multicolumn{2}{|c|}{ Signal Density } \\
\cline { 2 - 3 } & Male Urethra & Female Urethra \\
\hline PDE IA & + & $++(+)$ \\
PDE 2A & +++ & +++ \\
PDE 4A & + & +++ \\
PDE 4B & + & ++ \\
PDE 5A & ++ & ++ \\
PDE IOA & +++ & +++ \\
PDE IIA & + & +++ \\
\hline
\end{tabular}

Notes: Table displaying the semiquantitative expression of protein (characterized by distinct molecular weights) related to the PDE isoenzymes PDEIA (65 kDa), PDE2A ( $97 \mathrm{kDa})$, PDE4A/4B (66 kDa), PDE5A (89-99 kDa), PDEIOA (90/93 kDa) and PDEI IA $(65-95 \mathrm{kDa})$ in the human male and female urethra. $+=$ weak signal, $++=$ mediocre signal, +++ = dense signal.

supported by results from a study utilizing conventional immunohistochemistry in order to examine the presence of PDE isoenzymes in the male distal urethra. Immunosignals specific for PDE1A, 4A, 4B and 5A but not PDE2A were observed. Smooth muscle fibers positive for PDE 4B were seen transversed by nerves containing VIP, known to act as an endogenous stimulator of the cyclic AMP producing enzyme adenylyl cyclase, bundles of urethral smooth muscle immunoreactive for PDE5 were innervated by slender nerve fibers displaying the expression of CGRP. ${ }^{12}$ The expression of mRNA and its translation into PDE protein is only one part of the information required for the understanding of the role of this group of enzymes in urethral function, whereas functional studies are of paramount importance in order to draw substantial conclusions.

Given this premise, our observations are in accordance with the results from tissue bath studies using isolated human urethral tissue excised from females and males. The tonic contraction induced by an adrenergic agonist was reversed by the PDE5 inhibitors sildenafil and vardenafil, in isolated male urethral smooth muscle, the PDE4 inhibitor rolipram was shown to be most efficacious in reversing the adrenergic tension. ${ }^{10,11}$ It is believed that these experiments may provide evidence for a functional role of both PDE4 and PDE5 in the control of urethral smooth muscle tension. In vivo, rolipram has also been shown to enhance the relaxation of male mice urethra induced by the beta-3-adrenoreceptor agonist mirabegron, known to stimulate the production of the second messenger cyclic AMP. ${ }^{26}$ In cystomanometric studies in diabetic female rats, sildenafil partially reduced urethral pressure. $^{27}$

\section{Conclusion}

On the level of mRNA and function proteins, the expression of cyclic AMP and cyclic GMP PDE isoenzymes in both the human male and female urethra is not homogenous, different gender-specific patterns were observed. It remains to be established whether or not selective inhibition of PDE isoenzymes may represent a new option to selectively facilitate the relaxation of the outflow region in males and females.

\section{Abbreviations}

cDNA, complementary desoxyribonucleic acid; cyclic AMP/GMP, cyclic adenosine/guanosine monophosphate; $\mathrm{kDa}$, kilodalton; LUT, lower urinary tract; mRNA, messenger ribonucleic acid; NO, nitric oxide; NOS, nitric oxide synthase; PDE, phosphodiesterase enzyme; qRTPCR, quantitative reverse transcriptase polymerase chain reaction; USM, urethral smooth muscle; WB, Western blot.

\section{Disclosure}

The authors have no conflicts of interest in this work.

\section{References}

1. Barnea O, Gillon G. Model-based estimation of male urethral resistance and elasticity using pressure-flow data. Comput Biol Med. 2001;31:27-40. doi:10.1016/S0010-4825(00)00020-2

2. van der Werf BA, Creed KE. Mechanical properties and innervation of the smooth muscle layers of the urethra of greyhounds. Br J Urol Int. 2002;90:588-595. doi:10.1046/j.1464-410X.2002.02971.x

3. Mangera A, Osman NI, Chapple CR. Anatomy of the lower urinary tract. Surg Oxf Int Ed. 2013;31:319-325.

4. Livingston BP. Anatomy and neural control of the lower urinary tract and pelvic floor. Top Geriatr Rehabil. 2016;32:280-294. doi:10.1097/ TGR.0000000000000123

5. Andersson KE. Neurotransmission and drug effects in urethral smooth muscle. Scand J Uro Nephrol. 2001;207:26-34. doi:10.1080/003655901750174854

6. Hedlund P. Nitric oxide/cGMP-mediated effects in the outflow region of the lower urinary tract - Is there a basis for pharmacological targeting of cGMP? World J Urol. 2005;23:362-367. doi:10.1007/ s00345-005-0019-1

7. Küthe A, Mägert HJ, Ückert S, et al. Gene expression of the phosphodiesterases $3 \mathrm{~A}$ and $5 \mathrm{~A}$ in human corpus cavernosum penis. Eur Urol. 2000;38:108-114. doi:10.1159/000020262

8. Oger S, Behr-Roussel D, Gorny D, et al. Relaxation of phasic contractile activity of human detrusor strips by cyclic nucleotide phosphodiesterase type 4 inhibition. Eur Urol. 2007;51:772-781. doi:10.1016/j.eururo.2006.10.023

9. Ückert S, Oelke M. Phosphodiesterase (PDE) inhibitors in the treatment of lower urinary tract dysfunction. $\mathrm{Br} J$ Clin Pharmacol. 2011;72:197-204. doi:10.1111/j.1365-2125.2010.03828.x

10. Kedia GT, Oelke M, Sonnenberg JE, et al. Phosphodiesterase isoenzymes in the human urethra: a molecular biology and functional study. Eur $J$ Pharmacol. 2014;741:330-335. doi:10.1016/j. ejphar.2014.08.005 
11. Werkström V, Svensson A, Andersson KE, Hedlund P. Phosphodiesterase 5 in the female pig and human urethra: morphological and functional aspects. Br J Urol Int. 2006;98:414-423. doi:10.1111/j.1464-410X.2006.06217.x

12. Kedia GT, Ückert S, Oelke M, et al. Expression and distribution of phosphodiesterase isoenzymes in the human male urethra. Urology. 2015;85:964 e1-6. doi:10.1016/j.urology.2014.12.030

13. Lee JG, Moon D, Kang SH, et al. Relaxation effect of phosphodiesterase 5 inhibitor on the animal bladder and prostatic urethra: in vitro and in vivo study. Urol Int. 2010;84:231-235. doi:10.1159/ 000277604

14. Persson K, Andersson KE. Non-adrenergic, non-cholinergic relaxation and levels of cyclic nucleotides in rabbit lower urinary tract. Eur J Pharmacol. 1994;268:159-167. doi:10.1016/0922-4106(94) 90185-6

15. Tinel H, Stelte-Ludwig Hütter BJ, Sandner P. Pre-clinical evidence for the use of phosphodiesterase 5 inhibitors for treating benign prostatic hyperplasia and lower urinary tract symptoms. $\mathrm{Br} \mathrm{J} \mathrm{Urol}$ Int. 2006;98:1259-1263. doi:10.1111/j.1464-410X.2006.06501.x

16. Giuliano F, Ückert S, Maggi M, et al. The mechanism of action of phosphodiesterase type 5 (PDE5) inhibitors in the treatment of lower urinary tract symptoms related to benign prostatic hyperplasia. Eur Urol. 2013;63:506-516. doi:10.1016/j.eururo.2012.09.006

17. Ehren I, Iversen H, Jansson O, et al. Localization of nitric oxide synthase activity in the human lower urinary tract and its correlation with neuroeffector responses. Urology. 1994;44:683-687. doi:10.1016/S0090-4295(94)80206-8

18. Smet PJ, Jonavicius J, Marshall VR, de Vente J. Distribution of nitric oxide synthase immunoreactive nerves and identification of the cellular targets of nitric oxide in guinea-pig and human urinary bladder by cGMP immunohistochemistry. Neuroscience. 1996;71:337-348. doi:10.1016/0306-4522(95)00453-X

19. Barry CM, Ji E, Sharma H, et al. Morphological and neurochemical characteristics in female mice of reproductive age. Neurourol Urodyn. 2018;37:960-970. doi:10.1002/nau.23434
20. Fibbi B, Morelli A, Vignozzi L, et al. Characterization of phosphodiesterase type 5 expression and functional activity in the human male lower urinary tract. $J$ Sex Med. 2010;7(Pt 1):59-69. doi:10.1111/j.1743-6109.2009.01511.x

21. Truss MC, Ückert S, Stief CG, et al. Cyclic nucleotide phosphodiesterase (PDE) I soenzymes in the human detrusor smooth muscle. I. Identification and characterization. Urol Res. 1996;24:123-128. doi:10.1007/BF00304074

22. Ückert S, Sigl K, Waldkirch ES, et al. [Significance of phosphodiesterase isoenzymes in the control of human detrusor smooth muscle function. An immunohistochemical and functional study]. Urologe A. 2009;48:764-769. (Article in German). doi:10.1007/s00120-0091970-z.

23. Sonenberg N, Hinnebusch AG. Regulation of translation initiation in eukaryotes: mechanisms and biological targets. Cell. 2009;136:731-745. doi:10.1016/j.cell.2009.01.042

24. Ma XM, Blenis J. Molecular mechanisms of mTOR-mediated translational control. Nature Rev Mol Cell Biol. 2009;10:307-318. doi:10.1038/nrm2672

25. Ghazalpour A, Bennet B, Petyuk VA, et al. Comparative analysis of proteome and transcriptome variation in mouse. PloS Genetic. 2011;7:e1001393. doi:10.1371/journal.pgen.1001393

26. Alexandre EC, Kiguli LR, Calmasini FB, et al. Mirabegron relaxes urethral smooth muscle by dual mechanism involving b3-adrenoreceptor activation and alpha 1-adrenoreceptor blockade. Br J Pharmacol. 2016;173:415-428. doi:10.1111/bph.13367

27. Gomes de Souza Pegorare AB, Goncalves MA, Martiniano de Oliveira A, et al. Urethral dysfunction due to alloxan-induced diabetes. Urodynamic evaluation and action of sildenafil citrate. Acta Cir Bras. 2014;29:274-279. doi:10.1590/S0102-86502014000400009
Research and Reports in Urology

\section{Publish your work in this journal}

Research and Reports in Urology is an international, peer-reviewed, open access journal publishing original research, reports, editorials, reviews and commentaries on all aspects of adult and pediatric urology in the clinic and laboratory including the following topics: Pathology, pathophysiology of urological disease; Investigation and
Dovepress

treatment of urological disease; Pharmacology of drugs used for the treatment of urological disease. The manuscript management system is completely online and includes a very quick and fair peer-review system, which is all easy to use. Visit http://www.dovepress.com/ testimonials.php to read real quotes from published authors. 